\title{
ORIGINAL ARTICLE \\ Few genetic and environmental correlations between life history and stress resistance traits affect adaptation to fluctuating thermal regimes
}

\author{
T Manenti, JG Sørensen, NN Moghadam and V Loeschcke
}

Laboratory selection in thermal regimes that differed in the amplitude and the predictability of daily fluctuations had a marked effect on stress resistance and life history traits in Drosophila simulans. The observed evolutionary changes are expected to be the result of both direct and correlated responses to selection. Thus, a given trait might not evolve independently from other traits because of genetic correlations among these traits. Moreover, different test environments can induce novel genetic correlations because of the activation of environmentally dependent genes. To test whether and how genetic correlations among stress resistance and life history traits constrain evolutionary adaptation, we used three populations of $D$. simulans selected for 20 generations in constant, predictable and unpredictable daily fluctuating thermal regimes and tested each of these selected populations in the same three thermal regimes. We explored the relationship between genetic correlations between traits and the evolutionary potential of $D$. simulans by comparing genetic correlation matrices in flies selected and tested in different thermal test regimes. We observed genetic correlations mainly between productivity, body size, starvation and desiccation tolerance, suggesting that adaptation to the three thermal regimes was affected by correlations between these traits. We also found that the correlations between some traits such as body size and productivity or starvation tolerance and productivity were determined by test regime rather than selection regime that is expected to limit genetic adaptation to thermal regimes in these traits. The results of this study suggest that several traits and several environments are needed to explore adaptive responses, as genetic and environmentally induced correlations between traits as results obtained in one environment cannot be used to predict the response of the same population in another environment.

Heredity (2016) 117, 149-154; doi:10.1038/hdy.2016.34; published online 8 June 2016

\section{INTRODUCTION}

Temperature is one of the environmental measures that most strongly affects and limits the distribution of species, by affecting physiological processes, behavior, stress resistance and life history traits (Feder, 1988; Hoffmann and Parsons, 1991). An increasing number of evolutionary and ecological studies have recently focused on the effects of short- or long-term variation of temperatures (Ketola et al., 2004; Kingsolver et al., 2009; Hallsson and Bjorklund, 2012; Kellermann et al., 2015). Using fluctuating temperatures can be argued to have a greater ecological relevance compared with constant ones, as they are a better proxy of a natural environment (Boyce et al., 2006; Schreiber, 2010). Still, mechanisms that allow organisms to cope with and adapt to different fluctuating temperature regimes are not properly understood.

Short-term adaptive responses to varying temperatures are expected to depend on the ability to rapidly adjust the phenotype by phenotypic plasticity (Pal, 1998; Lande, 2009; Chevin and Lande, 2010; Kellermann et al., 2015). For instance, Manenti et al. (2014) found high levels of plasticity in a natural population of Drosophila simulans induced by different fluctuating thermal regimes that differed in the amplitude and predictability of daily fluctuations. A single generation exposure to fluctuating temperatures was shown to lead to an increased thermal stress resistance because of acclimation as induced by the experience of high and low temperatures during development (Hoffmann et al., 2003; Sejerkilde et al., 2003; Chown and Terblanche, 2007; Manenti et al., 2014). At the same time, coping with daily fluctuating temperatures is expected to demand higher energetic costs (trade-off), that may result in negative correlations between stress resistance and life history traits in flies exposed to fluctuating thermal regimes. However, after several generations of laboratory natural selection in constant or fluctuating thermal regimes, Manenti et al. (2015) found changes in the performance of flies in trait means but no changes in the levels of plasticity in body size, developmental time, desiccation and starvation tolerance as well as in heat and cold resistance. The authors suggested that plasticity might be evolutionary constrained and that evolutionary changes in trait means are the main adaptive mechanism to respond to constant, predictable and unpredictable daily fluctuations of temperatures. Similarly, Klepsatel et al. (2013) found similar levels of plasticity in several life history traits in six natural populations of Drosophila melanogaster from markedly different geographic locations, also suggesting that adaptation to such different thermal regimes might occur through changes in trait means rather than in the plasticity of these same traits. 
Trait means might, however, not be free to evolve independently from each other in different regimes because of genetic correlations among them. Genetic correlations can be the results of pleiotropy, linkage disequilibrium or of the correlated response of the two traits of interest with a third trait subjected to selection (Clark, 1987; Stearns, 1992). Changes in the environmental conditions can affect the variance of traits and trait interactions differently (Service and Rose, 1985; Hoffmann and Parsons, 1991; Hoffmann and Merila, 1999; Sgro and Hoffmann, 2004; Saltz, 2011), as the exposure to novel environmental conditions activate or depress the expression of different genes (Rose, 1984; Vieira et al., 2000; Mackay, 2001; Borevitz et al., 2002). Temperatures have been shown to have such effects on the genetic correlation between traits (Windig, 1994; Krebs and Loeschcke, 1999; de Jong and Imasheva, 2000; Norry and Loeschcke, 2002). For example, Norry and Loeschcke (2002) found that different temperatures and different genetic backgrounds affected the correlation between longevity and body size, and between body size and developmental time. The effect of the environmental conditions in shaping genetic constraints might affect the evolutionary response to different thermal regimes. Moreover, genetic interactions between traits (Hoffmann and Sgro, 2011; Duputie et al., 2012) can lead to indirect correlated responses observed in traits (Lande and Arnold, 1983) or limited response to selection in a nonstressful environment (Etterson and Shaw, 2001; Blows and Hoffmann, 2005; Kirkpatrick, 2009), affecting the evolutionary response to different thermal regimes.

As said before, Manenti et al. (2015) found that adaptation to constant, predictable and unpredictable daily fluctuations of temperatures was mainly achieved by evolutionary changes in trait means and not through changes in the levels of plasticity. Here we reanalyzed data from Manenti et al. (2015) to investigate how adaptation to constant, predictable and unpredictable thermal regimes affected the genetic correlations between four life history (productivity, egg-to-adult viability, developmental time and body size) and four stress resistance traits (heat, cold, starvation and desiccation tolerance) in flies maintained for 20 generations under natural laboratory selection in constant (C), predictable (PF) or unpredictable (UF) fluctuating thermal regimes. Moreover, we explored whether and how a single generation exposure to such different thermal regimes affected the correlations between traits (environmental correlation). Specifically, we investigated (1) the presence of genetic constraints among traits in flies selected for several generations in the C, PF and UF regimes. In the cases of significant correlations between traits, we hypothesized two possible scenarios: (a) correlations between traits are consistent in sign and magnitude between selection regimes, indicating that genetic correlations do not markedly shape adaptation to different thermal regimes; and (b) correlations between traits are different in different selection regimes, indicating an important role of genetic correlations in shaping adaptation to $\mathrm{C}, \mathrm{PF}$ and UF regimes.

We also investigated whether (2) a single generation exposure to different thermal regimes resulted in different genetic correlations between traits (environmental correlations). Traits environmentally correlated might limit the evolutionary potential of trait means, leading to similar environmental correlations between traits in flies selected in different selection regimes.

\section{MATERIALS AND METHODS}

The origin of the population

The population of D. simulans used in this study was collected close to Bologna, Italy (44.271647N, $11.04686 \mathrm{E}$ ), in August 2012 (Manenti et al., 2014). A mass population was established with $\sim 350$ wild-caught Drosophila females.
Flies were kept for two generations at $23^{\circ} \mathrm{C}$ before starting the laboratory natural selection (for further details, see Manenti et al., 2015).

\section{Selection procedure}

Flies were randomly divided into 9 groups (each group with $\sim 500$ individuals) that were randomly split in the three thermal selection regimes, with three biological replicates (replicate) for each of them. The three selection regimes were characterized by either daily constant $23^{\circ} \mathrm{C}$ (constant (C)), by consistent daily fluctuations of temperatures with the minimum and maximum temperatures of 13 and $28^{\circ} \mathrm{C}$, respectively (predictable fluctuating regime (PF)) and by a fluctuating temperature where the daily minimum and maximum temperatures were randomly generated (unpredictable fluctuating regime (UF)), without going below $13{ }^{\circ} \mathrm{C}$ or exceeding $28^{\circ} \mathrm{C}$, respectively. The 16 and $8 \mathrm{~h}$ of light and dark characterized the daily light cycle, respectively, and it was the same for all thermal regimes. The mean temperature in the three selection regimes was the same in the three selection regimes (for a graphical representation of the three selection regimes, see Manenti et al., 2015). Flies were selected in the three selection regimes for 20 generations before performing the phenotypic assays.

\section{Phenotypic assays}

After 20 generations of experimental evolution, 7 isofemale lines were established from each replicate within each selection regime. In order to achieve similar density of flies, for each isofemale line, groups of 40 eggs were transferred to vials with fresh food and randomly placed in the three thermal test regimes, leading to a total number of 189 isofemale lines (3 selection regimes $\times 3$ test regimes $\times 3$ replicate $\times 7$ isofemale lines). We assayed four stress resistance and four life history traits in the offspring of the same 21 isofemale lines of each selection regime. Ten randomly chosen female flies from each line were tested for each trait, except for egg-to-adult viability and developmental time. We decided to have the same isofemale line in all three test regimes at the same time, instead of applying a block design. We were afraid that the limited number of replication for each isofemale line (10 samples) could markedly increase the error within line if blocked. It is important to note that the same isofemale lines were assayed in all life history and stress resistance traits. For a more detailed description of the origin of flies and of the selection regimes, see Manenti et al. (2014).

Productivity. Productivity was assessed as the number of adult flies produced by a single female for 3 days from the age of 3 days in a 4-ml vial with standard oatmeal-sugar-yeast-agar medium. A total number of 1890 females was assayed.

Egg-to-adult viability and developmental time. Developmental time was the time taken by flies to complete the development, with time zero being defined as the time when the parental flies start to lay eggs. Egg-to-adult viability was measured as the ratio between the number of emerging flies out of 40 eggs. Egg-to-adult viability and developmental time were scored in three vials for each isofemale line, resulting in a total number of 22680 eggs being assayed.

Body size. Body size was assayed as centroid size based on 11 landmarks (Slice, 1996). The right wings were removed from 10 female flies of each line. The wings were dehydrated in ethanol and mounted on glass slides in lactic acid/ethanol (6:5). The coordinates of 11 landmarks were recorded for each wing by ImageJ 1.46r software (National Institute of Health, Bethesda, MD, USA). A total number of 1890 females was assayed.

Time to heat knockdown. Time to heat knockdown was measured as the time taken for flies to fall into a coma where they do not react to mechanic and light stimuli. A single fly was placed into a 5 -ml vial that was submerged into a water tank at $37.5^{\circ} \mathrm{C}$. A total number of 1890 females was assayed.

Chill coma recovery time. A single female was placed into a 5- $\mathrm{ml}$ vial that was submerged into a water tank at $0{ }^{\circ} \mathrm{C}$ for $3 \mathrm{~h}$. After this, flies were quickly moved to the laboratory at room temperature $\left(\mathrm{ca} .22 .5^{\circ} \mathrm{C}\right)$. The chill coma recovery time was recorded as the time until flies spontaneously recovered and they were able to stand on all legs. A total number of 1890 females was assayed. 
Desiccation. Desiccation tolerance was scored as the time flies could tolerate water deprivation before falling into a coma state, where flies did not react to an intensive light stimulus. A single female was placed into a 5-ml vial sealed with gauze to ensure equilibrium between the humidity inside and outside the vial. Vials were then put into an airtight tank with silica gel on the bottom, and with a relative humidity close to $0 \%$. A total number of 1890 females was assayed.

Starvation. Starvation tolerance was measured as the time flies could tolerate food deprivation. In order to avoid dehydration of flies during the test, flies were kept in $7 \mathrm{ml}$ vials containing $2 \mathrm{ml}$ of agar-water solution. The number of flies that did not respond to external stimuli was scored every $8 \mathrm{~h}$. A total number of 1890 females was assayed.

\section{Statistical analysis}

In the present study we reanalyzed the data originally collected by Manenti et al. (2015), who studied the effect of different thermal selection and test regimes on several life history and stress resistance traits. The present study aims to investigate whether the adaptation to different thermal regimes can be affected by genetic correlations between traits.

The analyses were divided in two parts. In the first section, the variancecovariance matrix ( $G$ matrix) was calculated in order to quantify the additive genetic variation of each trait in each selection and test regime, following a halfsib genetic model. Differences between replicates were tested for each trait by linear mixed model (Bates et al., 2014) with selection and test regimes treated as fixed effects and with replicate and isofemale line nested in replicate treated as random effects. We found a nonsignificant effect of replicate in our model and therefore removed the factor replicate from our model (data not shown).

In order to compare $\mathrm{G}$ matrices in different selection and test regimes we estimated pseudovalues of (co)variances by the 'jack-knife method' (Knapp et al., 1989; Fox et al., 1999; Roff, 2002). This method allowed us to get multiple estimates of correlation between the same two traits by eliminating in turn one isofemale line ( $j$ th line) and then calculating pseudovalues of (co)variances between pairs of traits based on the remaining 20 isofemale lines. This led to 21 estimates of (co)variances within selection and test regimes. We decided not to apply logarithmic transformations on the variances obtained, as eventual negative logarithms affect the calculation of covariances. However, simulations made using the 'jack-knife method' to estimate genetic correlations showed that this method also worked properly with untransformed data (Roff and Preziosi, 1994; Simons and Roff, 1994). To reduce the complexity of the data and to correct for eventual not-independency between estimates of (co)variances, we applied principal component analysis. The loadings of each principal component were used as response variables for the multivariate analysis of variance, whereas selection regime, test regime and the interaction between these two factors were the explanatory variables. $P$-values associated with the explanatory variables were obtained by a randomization process. We stored the approximated F-ratio of each explanatory variable (observed F-ratio). Then, we randomly assigned the different isofemale lines among selection and test regimes (repeated 10000 times) and applied the same statistics to obtain a distribution of approximated F-ratios for the randomized populations. The probability of getting an approximated F-ratio for the randomized populations larger than the observed F-ratio was calculated as $(n+1) /(10000+1)$, where $n$ is the number of times where the randomized F-ratio $>$ observed F-ratio. For more detailed information on the statistics applied, see Roff (2002).

In the second part of the analysis, we looked at the distribution of genetic correlations between traits. This descriptive analysis aims at identifying which traits correlated the most and to visualize the difference between selection and test regimes found with multivariate analyses described above. For each selection and test regime we calculated Pearson's correlation coefficients $(r)$ between pairs of traits based on the mean of 21 isofemale lines. We used false discovery rate to correct for multiple test comparisons, with a threshold for significance of $P<0.001$. The analyses were performed in $\mathrm{R}$ ( $\mathrm{R}$ Core Team, 2015).

\section{RESULTS}

Both selection and test regimes affected the distribution of genetic correlations between traits (Table 1). Differences between selection
Table 1 Results from the multivariate analysis of variance (MANOVA) on the effect of selection regime, test regime and their interaction on the genetic correlations between traits

\begin{tabular}{lccc}
\hline Source of variation & Pillai's trace & $F$ & $P$ \\
\hline Selection & $0.084_{(2)}$ & $8.6_{(14,2716)}$ & $<0.01$ \\
Test & $0.083_{(2)}$ & $8.4_{(14,2716)}$ & $<0.001$ \\
Selection $\times$ test & $0.25_{(4)}$ & $13_{(28,2716)}$ & 0.66 \\
\hline
\end{tabular}

For each selection and test regime we calculated multiple estimates of correlations between pairs of traits by the 'jack-knife method'. We then ran principal component analysis (PCA) on genetic correlations between traits. The loadings of each principal component were used as response variables for the MANOVA. The MANOVA provided estimates of Pillai's trace and approximated F-values (F). Probability associated with the different sources of variations was obtained by a randomization process. $P$ is probability associated with F-value. Degrees of obtained by a randomization process. $P$ is
freedom are given between parentheses.

regimes demonstrated that adaptation to different thermal regimes might occur through specific correlations between traits. The significant differences between test regimes indicate that genetic correlations between traits are environmentally specific. The interaction between selection and test regimes was nonsignificant, indicating that the three genetic correlations in flies from different selection regimes were affected by test regimes in a similar way (Table 1 ).

The genetic correlation coefficients among all traits for each selection and test regime are reported in Table 2. Among all selection and test regimes we observed 15 significant out of 189 tested correlations after multiple testing corrections. The nine matrices showed that significant correlations were found mainly between four traits, starvation tolerance, desiccation tolerance, body size and productivity. The largest number of correlations was observed in flies selected in the PF regime (eight) followed by flies selected in the UF regime (five), whereas two genetic correlations were observed in flies selected in the $\mathrm{C}$ regime (Table 2). Looking at the three test regimes, we observed one correlation in the $\mathrm{C}$ test regime, six in the PF test regime and eight in the UF test regime.

Flies selected in the $\mathrm{C}$ regime showed significant correlations between starvation tolerance and time to heat knockdown and between body size and productivity only when tested in the UF regime. Flies selected in the PF regime showed a negative correlation between starvation tolerance and productivity when tested in the $\mathrm{C}$ regime, between starvation tolerance and productivity, desiccation tolerance and productivity, starvation and desiccation tolerance and between developmental time and body size when tested in the PF regime, and between starvation tolerance and productivity, body size and productivity and between body size and starvation tolerance when tested in the UF regime. Flies selected in the UF regime showed negative correlations between starvation tolerance and productivity, desiccation tolerance and time to heat knockdown when tested in the PF regime, and between starvation tolerance and productivity, desiccation tolerance and time to heat knockdown and between body size and productivity when tested in the UF regime (Table 2).

\section{DISCUSSION}

Laboratory natural selection in daily constant, predictable and unpredictable fluctuating thermal regimes affected the mean performance in stress resistance and life history traits of flies indicating adaptation to thermal regimes (Manenti et al., 2015). Here we investigated the underlying genetic correlations between traits in different selection and test regimes. We tested whether genetic correlations between traits may interfere with evolutionary changes. We investigated (1) the presence of genetic correlations among several life history and stress resistance traits. Such correlations might be 


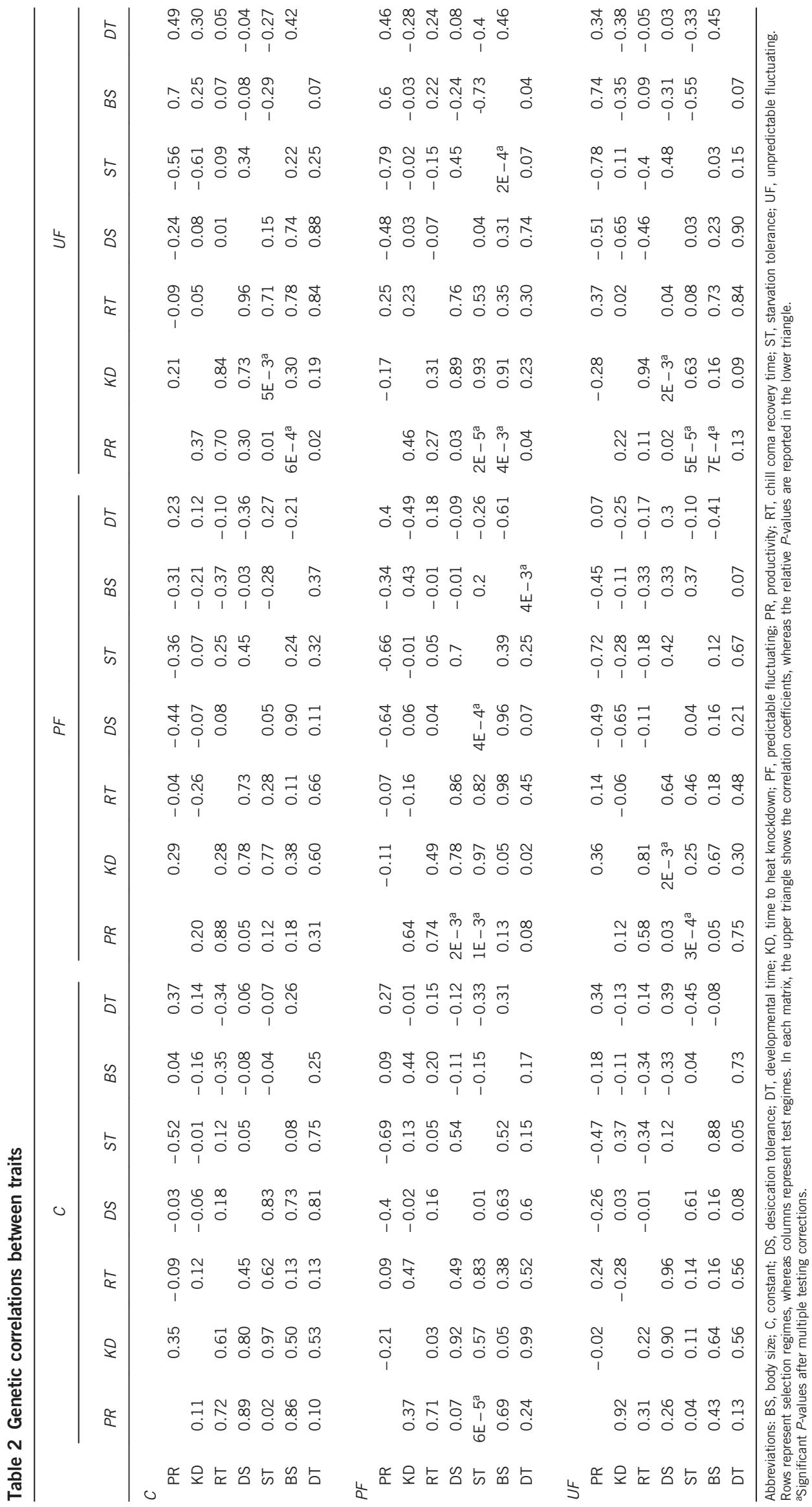


similar (a) or different (b) in flies selected in the different thermal regimes. Furthermore, we investigated (2) whether test regimes affected the correlations between traits in a nonadditive way (environmental correlations), resulting in different genetic correlations within selection regimes in different test regimes.

We found that both selection and test regimes have a marked effect on shaping the correlations between traits, with no interaction between selection and test regimes. We observed 15 significant correlations out of 189 between trait correlations tested. Although this number is small, the correlations were consistently observed between productivity, body size, starvation and desiccation tolerance. A previous study of the genetic correlation between traits, using the same three test regimes as in this study, found no significant correlations between any traits in the base population (Manenti et al., 2014). This suggests that genetic correlations evolved under the three thermal regimes (scenario 1-b).

A negative correlation between starvation tolerance and productivity was observed among flies selected and tested in the two fluctuating regimes, indicating that more productive flies generally showed a poor starvation resistance. Thus, a higher number of genetic correlations was observed in flies selected in the two fluctuating regimes as compared with flies selected in the constant regime. This trade-off might be explained by the competitive allocation of the limited resources into reproduction or into starvation tolerance (Stearns, 1989). The preference of allocating the resources to egg laying rather than to starvation tolerance can be the result of higher evolutionary relevance of the egg laying compared with starvation tolerance (Flatt, 2011). Furthermore, flies selected in the PF regime and tested in the UF regime showed a negative correlation between starvation tolerance and body size. In these two regimes, lines with larger flies laid more eggs and were less starvation tolerant as compared with lines with smaller flies. However, when trait means were assessed, we found higher starvation tolerance and larger body size in flies selected in the $\mathrm{PF}$ and UF regimes compared with flies selected in the $\mathrm{C}$ regime. Thus, even if the results in trait means might indicate that larger flies could better tolerate food deprivation, the negative genetic correlation between these traits points in the opposite direction. The trade-offs between body size and starvation tolerance were different depending on whether we investigated the physiological costs at the intraindividual or the population level (Flatt, 2005). Within isofemale lines individuals shared a similar genetic background, resulting in an expected fixed genetic correlation between traits. Thus, trade-offs observed within isofemale lines do not contribute to the evolutionary response expected at the population level (Flatt and Kawecki, 2007).

We hypothesized that if correlations changed depending on the test regimes (environmental correlations), we would observe different correlations between traits in different test regimes (Service and Rose, 1985; de Jong, 1990). We found that test regimes significantly affected the genetic correlations between traits, indicating a strong effect of test regime on the performance of flies (Hoffmann et al., 2003; Chown and Terblanche, 2007; Manenti et al., 2014, 2015). The lower number of correlations observed in the $\mathrm{C}$ regime compared with both fluctuating regimes might be the results of more benign conditions experienced by flies in this regime compared with the two fluctuating regimes, as suggested earlier (Manenti et al., 2014). The correlations observed between starvation tolerance and productivity in PF selected flies, and between desiccation tolerance and heat resistance in flies selected in the UF regime, were similar across test regimes. This indicates that the genetic correlations between these traits were determined by the selection regimes and were independent of the test regimes, as indicated by the nonsignificant interaction between selection and test regimes in the multivariate analysis of variance. However, for some other pairs of traits such as productivity and body size as well as starvation tolerance and productivity, we observed similar correlations when flies from all three selection regimes were tested in the UF regime. Contrary to what was said above, here it seems that the test regime determined the correlation between these traits. Correlations determined by the test environment might limit the potential for these traits to evolve in response to selection in these three thermal regimes. How strong the limitation in the evolution of traits is due to genetic correlations is not easy to quantify. A study showed that despite genetic correlations between morphological features of butterfly wings, independent evolution of the same morphological traits was still possible (Beldade and Brakefield, 2002), suggesting a weak effect of genetic correlations on the evolution of these particular traits.

In summary, the results of this study show that correlations between traits may play an important role for the adaptation to varying environments. In particular, we showed that certain pairs of traits such as productivity and starvation tolerance as well as heat resistance and desiccation tolerance might interact enhancing adaptation to different thermal regimes. However, correlations between other pairs of traits such as body size and productivity or starvation tolerance and productivity were determined by test regime that can limit the evolution of these traits (Sgro and Hoffmann, 2004). The results of this study also suggest that it is important assaying several traits and several environments to explore adaptation. Genetic and environmental correlations affect adaptation and they might lead to different outcomes, and results obtained in one environment cannot be considered fully informative regarding the response expected by the same population in another environment.

\section{DATA ARCHIVING}

Data available from the Dryad Digital Repository: http://dx.doi.org/ 10.5061/dryad.hf887.

\section{CONFLICT OF INTEREST}

The authors declare no conflict of interest.

\section{ACKNOWLEDGEMENTS}

We thank Doth Andersen, Annemarie Højmark and Marta Falaschi for their great help in the laboratory, Sandro Cavicchi for the collection of the wild population and Mads Fristrup Schou for assistance in programming in R. The study was supported by the Graduate School of Science and Technology (GSST) at Aarhus University, by a Sapere Aude: DFF-Starting Grant (to JGS) and a large frame grant (to VL) from the Danish Council for Independent Research, Natural Sciences (DFF 4002-00113B).

Bates D, Maechler M, Walker S (2014). Ime4: Linear Mixed-Effects Models Using Eigen and S4. $R$ packages version 1.1-5. Available from http://cran.r-project.org/package $=$ Ime4.

Beldade P, Brakefield PM (2002). The genetics and evo-devo of butterfly wing patterns. Nat Rev Genet 3: 442-452.

Blows MW, Hoffmann AA (2005). A reassessment of genetic limits to evolutionary change. Ecology 86: 1371-1384.

Borevitz JO, Maloof JN, Lutes J, Dabi T, Redfern JL, Trainer GT et al. (2002). Quantitative trait loci controlling light and hormone response in two accessions of Arabidopsis thaliana Genetics 160: 683-696.

Boyce MS, Haridas CV, Lee CT, Demography NS (2006). Demography in an increasingly variable world. Trends Ecol Evol 21: 141-148.

Chevin LM, Lande R (2010). When do adaptive plasticity and genetic evolution prevent extinction of a density-regulated population? Evolution 64: 1143-1150.

Chown SL, Terblanche JS (2007). Physiological diversity in insects: ecological and evolutionary contexts. Adv In Insect Phys 33: 50-152. 
Clark AG (1987). Senescence and thegenetic-correlation hang-up. Am Nat 129: 933-940. de Jong G (1990). Quantitative genetics of reaction norms. J Evol Biol 3: 447-468.

de Jong G, Imasheva A (2000). Genetic variance in temperature dependent adult size deriving from physiological genetic variation at temperature boundaries. Genetica 110 : 195-207.

Duputie A, Massol F, Chuine I, Kirkpatrick M, Ronce $O$ (2012). How do genetic correlations affect species range shifts in a changing environment? Ecol Lett 15: 251-259.

Etterson JR, Shaw RG (2001). Constraint to adaptive evolution in response to global warming. Science 294: 151-154.

Feder ME (1988). Temperature biology of animals. Science 241: 1108-1108.

Flatt T (2005). The evolutionary genetics of canalization. Q Rev Biol 80: 287-316.

Flatt T (2011). Survival costs of reproduction in Drosophila. Exp Gerontol 46: 369-375

Flatt T, Kawecki TJ (2007). Juvenile hormone as a regulator of the trade-off between reproduction and life span in Drosophila melanogaster. Evolution 61: 1980-1991.

Fox CW, Czesak ME, Mousseau TA, Roff DA (1999). The evolutionary genetics of an adaptive maternal effect: egg size plasticity in a seed beetle. Evolution 53: 552-560.

Hallsson LR, Bjorklund M (2012). Selection in a fluctuating environment leads to decreased genetic variation and facilitates the evolution of phenotypic plasticity. J Evol Biol 25: 1275-1290.

Hoffmann AA, Merila J (1999). Heritable variation and evolution under favourable and unfavourable conditions. Trends Ecol Evol 14: 96-101.

Hoffmann AA, Parsons PA (1991). Evolutionary Genetics and Environmental Stress. Oxford University Press: Oxford.

Hoffmann AA, Sgro CM (2011). Climate change and evolutionary adaptation. Nature 470: $479-485$.

Hoffmann AA, Sørensen JG, Loeschcke V (2003). Adaptation of Drosophila to temperature extremes: bringing together quantitative and molecular approaches. J Therm Biol $\mathbf{2 8}$ $175-216$.

Kellermann V, Hoffmann AA, Kristensen TN, Moghadam NN, Loeschcke V (2015). Experimental evolution under fluctuating thermal conditions does not reproduce patterns of adaptive clinal differentiation in Drosophila melanogaster. Am Nat 186: 582-593.

Ketola T, Laakso J, Kaitala V, Airaksinen S (2004). Evolution of Hsp90 expression in Tetrahymena thermophila (Protozoa, Ciliata) populations exposed to thermally variable environments. Evolution 58: 741-748.

Kingsolver JG, Ragland GJ, Diamond SE (2009). Evolution in a constant environment thermal fluctuations and thermal sensitivity of laboratory and field populations of Manduca sexta. Evolution 63: 537-541.

Kirkpatrick M (2009). Patterns of quantitative genetic variation in multiple dimensions. Genetica 136: 271-284.

Klepsatel P, Galikova M, De Maio N, Huber CD, Schlotterer C, Flatt T (2013). Variation in thermal performance and reaction norms among populations of Drosophila Melanogaster. Evolution 67: 3573-3587.

Knapp SJ, Bridges-Jr WC, Yang MH (1989). Nonparametric confidence interval estimators for heritability and expected selection response. Genetics 121: 891-899.
Krebs RA, Loeschcke V (1999). A genetic analysis of the relationship between life-history variation and heat-shock tolerance in Drosophila buzzatii. Heredity 83: 46-53.

Lande R (2009). Adaptation to an extraordinary environment by evolution of phenotypic plasticity and genetic assimilation. J Evol Biol 22: 1435-1446.

Lande R, Arnold SJ (1983). The measurement of selection on correlated characters. Evolution 37: 1210-1226.

Mackay TFC (2001). The genetic architecture of quantitative traits. Annu Rev Genet 35: 303-339.

Manenti T, Loeschcke V, Moghadam NN, Sørensen JG (2015). Phenotypic plasticity is not affected by experimental evolution in constant, predictable or unpredictable fluctuating thermal environments. J Evol Biol 28: 2078-2087.

Manenti T, Sørensen JG, Moghadam NN, Loeschcke V (2014). Predictability rather than amplitude of temperature fluctuations determines stress resistance in a natural population of Drosophila simulans. J Evol Biol 27: 2113-2122.

Norry FM, Loeschcke V (2002). Temperature-induced shifts in associations of longevity with body size in Drosophila melanogaster. Evolution 56: 299-306.

Pal C (1998). Plasticity, memory and the adaptive landscape of the genotype. Proc $R$ Soc $B$ Biol Sci 265: 1319-1323.

R Core Team (2015). A Language and Environment for Statistical Computing. R Foundation for Statistical Computing: Vienna, Austria.

Roff D (2002). Comparing G matrices: a MANOVA approach. Evolution 56: 1286-1291.

Roff DA, Preziosi R (1994). The estimation of the genetic correlation - the use of the Jackknife. Heredity 73: 544-548.

Rose MR (1984). Genetic covariation in drosophila life-history - untangling the data. Am Nat 123: 565-569.

Saltz JB (2011). Natural genetic variation in social environment choice: context-dependent gene-environment correlation in Drosophila melanogaster. Evolution 65: 2325-2334.

Schreiber SJ (2010). Interactive effects of temporal correlations, spatial heterogeneity and dispersal on population persistence. Proc R Soc B Biol Sci 277: 1907-1914.

Sejerkilde M, Sørensen JG, Loeschcke V (2003). Effects of cold and heat hardening on thermal resistance in Drosophila melanogaster. J Insect Physiol 49: 719-726.

Service PM, Rose MR (1985). Genetic covariation among life-history components - the effect of novel environments. Evolution 39: 943-945.

Sgro CM, Hoffmann AA (2004). Genetic correlations, tradeoffs and environmental variation. Heredity 93: 241-248.

Simons AM, Roff DA (1994). The effect of environmental variability on the heritabilities of traits of a field cricket. Evolution 48: 1637-1649.

Slice DE (1996). Introduction to Landmark Methods. Plenum Press: New York.

Stearns SC (1989). Trade-offs in life-history evolution. Funct Ecol 3: 259-268.

Stearns SC (1992). The Evolution of Life Histories. Oxford University Press: Oxford.

Vieira C, Pasyukova EG, Zeng ZB, Hackett JB, Lyman RF, Mackay TF (2000). Genotypeenvironment interaction for quantitative trait loci affecting life span in Drosophila melanogaster. Genetics 154: 213-227.

Windig JJ (1994). Genetic correlations and reaction norms in wing pattern of the tropical butterfly bicyclus-anynana. Heredity 73: 459-470. 